\title{
In situ tissue regeneration using a novel tissue-engineered, small-caliber vascular graft without cell seeding
}

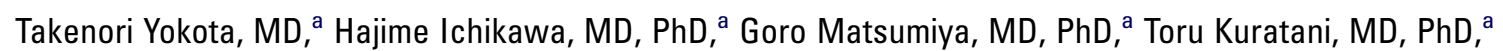

Taichi Sakaguchi, MD, PhD, ${ }^{\text {a }}$ Shigemitsu Iwai, MD, PhD, ${ }^{a}$ Yukitoshi Shirakawa, MD, PhD, ${ }^{a}$ Kei Torikai, MD, PhD, Atsuhiro Saito, $\mathrm{PhD},{ }^{a}$ Eiichiro Uchimura, $\mathrm{PhD},{ }^{a}$ Naomasa Kawaguchi, $\mathrm{PhD},{ }^{\mathrm{b}}$ Nariaki Matsuura, MD, $\mathrm{PhD},{ }^{\mathrm{b}}$ and Yoshiki Sawa, MD, PhD ${ }^{\mathrm{a}}$

From the Department of Cardiovascular Surgery, Osaka University Graduate School of Medicine, ${ }^{\text {a }}$ Osaka, Japan; and Department of Pathology, Osaka University School of Allied Health Science, ${ }^{b}$ Osaka, Japan.

Received for publication Nov 13, 2007; revisions received Jan 7, 2008; accepted for publication Feb 4, 2008.

Address for reprints: Yoshiki Sawa, MD $\mathrm{PhD}, 2$-2 Yamada-oka, Suita, Osaka 5650871, Japan (E-mail: sawa@surg1.med. osaka-u.ac.jp)

J Thorac Cardiovasc Surg 2008;136:900-7

$0022-5223 / \$ 34.00$

Copyright (C) 2008 by The American Association for Thoracic Surgery

doi:10.1016/j.jtcvs. 2008.02 .058
Objective: Various types of natural and synthetic scaffolds with arterial tissue cells or differentiated stem cells have recently attracted interest as potential small-caliber vascular grafts. It was thought that the synthetic graft with the potential to promote autologous tissue regeneration without any seeding would be more practical than a seeded graft. In this study, we investigated in situ tissue regeneration in small-diameter arteries using a novel tissue-engineered biodegradable vascular graft that did not require ex vivo cell seeding.

Methods: Small-caliber vascular grafts (4 $\mathrm{mm}$ in diameter) were fabricated by compounding a collagen microsponge with a biodegradable woven polymer tube that was constructed in a plain weave pattern with a double layer of polyglycolic acid (core) and poly-L-lactic acid (sheath) fibers. We implanted these tissue-engineered vascular grafts bilaterally into the carotid arteries of mongrel dogs (body weight, 20-25 kg). No anticoagulation regimen was used after implantation. We sacrificed the dogs $2,4,6$, and 12 months ( $n=4$ in each group) after implantation and evaluated the explants histologically and biochemically.

Results: All of the tissue-engineered vascular grafts were patent with no signs of thrombosis or aneurysm at any time. Histologic and biochemical examinations showed excellent in situ tissue regeneration with an endothelial cell monolayer, smooth muscle cells, and a reconstructed vessel wall with elastin and collagen fibers.

Conclusion: Our study indicated that this novel tissue-engineered vascular graft promoted in situ tissue regeneration and did not require ex vivo cell seeding, thereby conferring better patency on small-caliber vascular prostheses.

I $\mathrm{n}$ the area of vascular surgery, vascular prostheses prepared from expanded polytetrafluoroethylene (ePTFE) or Dacron are clinically used for reconstructing large-diameter arteries, such as the aorta and iliac artery. However, these synthetic materials are clinically unacceptable for reconstructing small-diameter arteries (ie, inner diameter of $<5 \mathrm{~mm}$ ), which are required in lower-extremity bypass and coronary artery bypass grafting procedures, because they are foreign bodies and thrombosis can occur on the luminal surface, resulting in occlusion. For such small artery bypass grafting procedures, autologous arterial (eg, internal thoracic artery and radial artery) or venous (eg, saphenous vein) grafts still remain the most ideal vascular substitutes. However, they cannot always be used because of their poor quality and inadequate size or length. Moreover, a second surgical procedure is required to obtain the vessel. Thus, it is imperative to develop innovative technologies targeting the fabrication of small-caliber vascular grafts.

The concept of tissue engineering using biodegradable materials was recently proposed, ${ }^{1}$ and such materials have been developed and clinically applied with autologous cell seeding or bioreactor culturing. ${ }^{2-9}$ However, the usefulness of these materials for 


\author{
Abbreviations and Acronyms \\ $\mathrm{ECM}=$ extracellular matrix \\ ePTFE $=$ expanded polytetrafluoroethylene \\ PGA $=$ polyglycolic acid \\ PLLA = poly-L-lactic acid \\ TEVG $=$ tissue-engineered vascular graft
}

reconstruction of small-caliber vascular grafts has not been demonstrated. Moreover, pretreatment with autologous cell seeding or bioreactor culturing can be complicated and invasive, and will render the graft prone to infection. We previously developed a tissue-engineered patch prepared from biodegradable polymer with collagen microsponge that did not require pretreatment and showed good in situ tissue regeneration of the autologous vessel tissue in both venous and arterial grafts. ${ }^{10}$

In the present study, we developed a novel tissueengineered small-caliber vascular graft that did not require pretreatment with cell seeding and promoted autologous tissue regeneration by inducing the proliferation and differentiation of endothelial and smooth muscle progenitor cells after implantation. The objective of this study was to investigate the biological performance and biochemical characteristics of this graft.

\section{Materials and Methods Scaffold Design}

A newly designed small-caliber vascular graft (4 $\mathrm{mm}$ in diameter) was fabricated by compounding a collagen microsponge with a cylindrically woven biodegradable and absorbable polymer tube that was constructed using core-sheath compound yarn with polyglycolic acid (PGA) and poly-L-lactic acid (PLLA) fibers (Figure 1, $A$ and $B$ ). The core-sheath yarn was fabricated by air-jet spinning technology using a core thread composed of 30 PLLA fibers (each having a diameter of $20 \mu \mathrm{m}$ ) and a sheath thread composed of 56 PGA fibers (each having a diameter of $15 \mu \mathrm{m}$ ). The coresheath yarn thus created had a porous 3-dimensional structure. All of these materials are biocompatible and biodegradable, and have received Food and Drug Administration clearance. These tissueengineered vascular grafts (TEVGs) were provided by Senko Medical Instrument Manufacturing Co Ltd (Tokyo, Japan). In addition, TEVGs with the collagen microsponge were prepared using bovine type I collagen. The techniques for fabricating the collagen microsponge are described in detail by Chen. ${ }^{11}$ Briefly, the PGA/PLLA tube graft was immersed in a solution containing type I collagen (Koken Co Ltd, Tokyo, Japan), frozen at $-80^{\circ} \mathrm{C}$ for 12 hours, and lyophilized in a vacuum of $0.2 \mathrm{~mm} \mathrm{Hg}$ for an additional 24 hours to allow the formation of the collagen microsponge. The collagen microsponge was further cross-linked by treatment with glutaraldehyde vapor through exposure to a $25 \%$ glutaraldehyde aqueous solution at $37^{\circ} \mathrm{C}$ for 4 hours. The graft structure and collagen microsponge are thought to have good cell attachment properties, and they promote in situ tissue regeneration because of their porous 3-dimensional structures. The scanning electron micrographs of the double-layered thread structure and graft surface are shown in Figure $1, C, D$, and $E$.

\section{In Vitro Study of Cell Culture Growth}

To verify the cellular affinity and proliferative ability of the collagen microsponge, murine fibroblasts (NIH3T3; American Type Culture Collection, Manassas, Va) and human umbilical vascular endothelial cells (Cambrex, East Rutheford, NJ) were seeded at a cell density of 300 cells $/ \mathrm{mm}^{2}$ onto the 2 sets of TEVGs with the collagen microsponge $(n=6)$ or without it $(n=6)$. After culture for 3 days in the routine manner, the surfaces of TEVGs were observed by scanning electron microscopy, and the number of cells attached to the graft was counted by a water-soluble tetrazolium assay (Cell Counting Kit; Dojindo Chemicals, Japan).

\section{In Vivo Study of Implantation in Mongrel Dogs}

We used 24 mongrel dogs (weight, 20-25 kg) in this study. All animals were anesthetized by intramuscular injection of $10 \mathrm{mg} / \mathrm{kg}$ ketamine (Sankyo Co, Tokyo, Japan) and $2 \mathrm{mg} / \mathrm{kg}$ of xylazine (Bayer Medical Co, Tokyo, Japan) followed by intubation and mechanical ventilation. An adequate level of anesthesia was maintained by intravenous continuous infusion of propofol (AstraZeneca, Osaka, Japan) at a rate of $4 \mathrm{mg} / \mathrm{kg} / \mathrm{h}$ throughout the operative procedure and additional injections of ketamine $(5 \mathrm{mg} / \mathrm{kg} / \mathrm{h})$. Bilateral carotid artery grafting was performed using TEVGs (4 $\mathrm{mm}$ in diameter, 30 $\mathrm{mm}$ in length). The bilateral carotid arteries were carefully exposed through a midline neck incision, and $100 \mathrm{IU} / \mathrm{kg}$ of heparin was administered intravenously before vascular clamping. The grafts were interposed in the carotid arteries, and proximal and distal end-to-end anastomoses were performed with continuous sutures of 7-0 monofilament polypropylene. All surgeries were successful, and there was no significant bleeding from the vascular graft after declamping the carotid arteries. Finally, protamine was administered intravenously, and the neck incision was closed. Antibiotics were given intravenously during surgery, but anticoagulants or antiplatelets were not administered postoperatively. After the surgical procedure was completed, the animal was extubated and resumed normal activities. At $2,4,6$, and 12 months after implantation ( $\mathrm{n}=4$ in each group), the animals were anesthetized again by the same method as above. The neck incision was reopened, the surgical site was examined for adhesions and fibrosis, and the implanted graft was carefully exposed. The animal was euthanized, and the grafts were carefully explanted and processed immediately or frozen for morphologic, histologic, immunohistochemical, and biochemical examinations. As the control, 8 ePTFE grafts of the same size were implanted in the same manner and explanted 2, 4, 6, and 12 months after implantation (n $=2$ in each group). The investigation conforms to the Principles of Laboratory Animal Care, formulated by the National Society for Medical Research, and the Guide for the Care and Use of Laboratory Animals, published by the National Academy of Science and the National Institutes of Health (Publication No. 86-23, revised 1996).

\section{Morphologic Examination}

The explanted grafts were incised longitudinally and flattened with their luminal side facing upward. The lumina were photographed in detail after gross examination. The middle portions of specimens were prepared in a typical fashion for scanning electron microscopy 

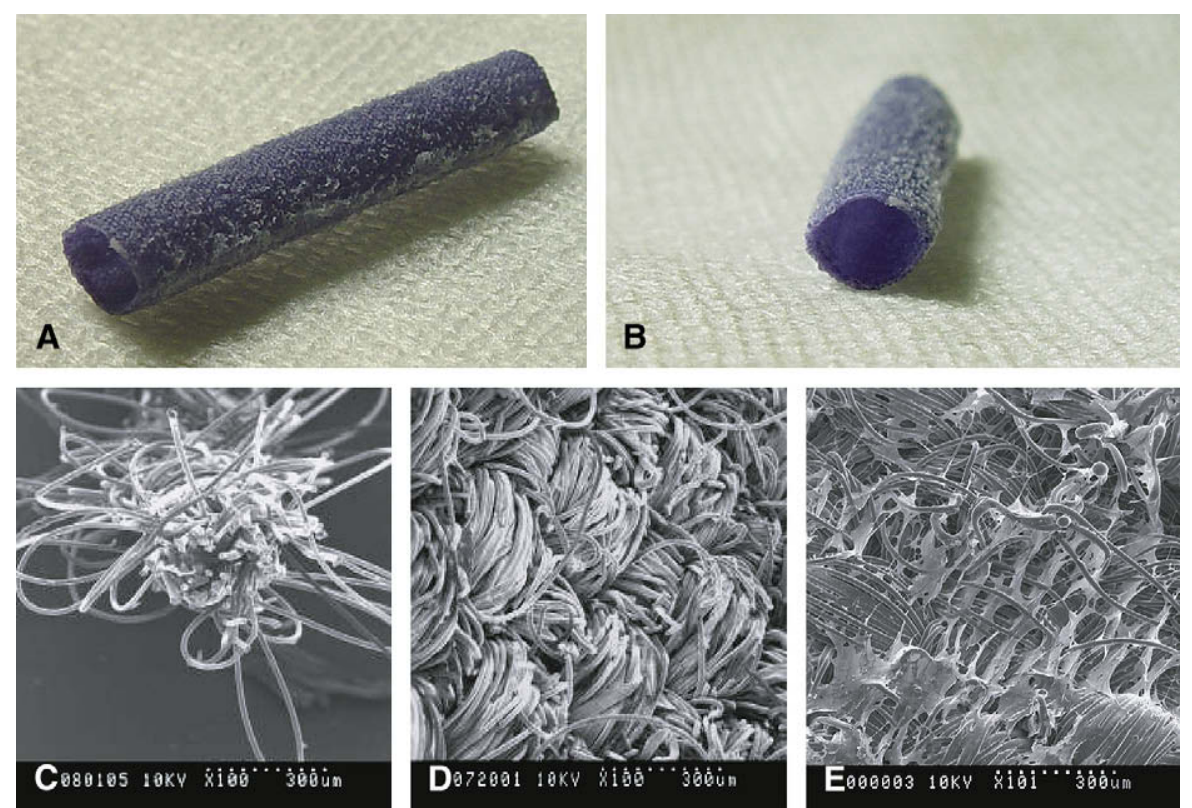

Figure 1. The newly designed smalldiameter vascular graft (A, B) was fabricated using a biodegradable woven polymer tube constructed in a plain weave pattern using a double-layered thread with PLLA fibers as the core and PGA fibers as the sheath. Scanning electron micrograph of the double-layered thread (C). The graft surface shows the porous 3-dimensional structure (D) and interconnected pore structure after incorporation of the collagen microsponge (E) (magnification, 100 $\times$ ).

and were observed with a scanning electron microscope (Model S-800; Hitachi, Tokyo, Japan).

\section{Histologic and Immunohistologic Examination}

Longitudinal samples of the explanted grafts were fixed in $10 \%$ buffered formalin and embedded in paraffin. Each cross-section that included the native tissue proximal and distal to TEVGs was examined by double staining with hematoxylin-eosin and Victoria blue, and with elastica van Gieson stain. Immunohistologic staining was also performed to identify the cell types in the explanted tissue specimens of the vascular grafts. The antibodies for immunohistochemistry included monoclonal antibody to $\alpha$-smooth muscle actin (clone 1A4; DAKO, Carpenteria, Calif), and polyclonal antibody to von Willebrand factor (Cat N: N1505, DAKO).

\section{Biochemical Examination}

Collagen formation in the explanted grafts was quantified to evaluate the degree of regeneration of the autologous tissue. The hydroxyproline content of the lyophilized samples was determined as an indicator of the collagen content using a modification of the assay described by Huzer
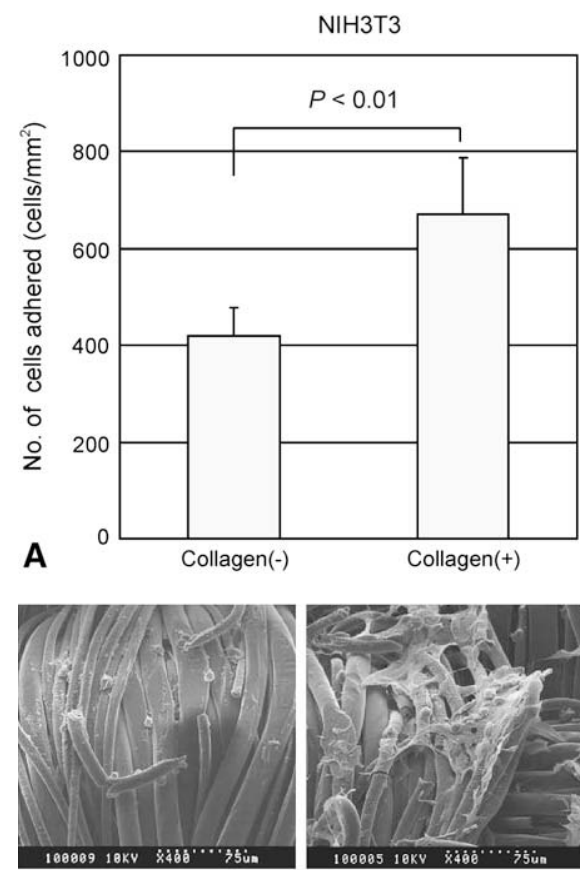

C Collagen(-)

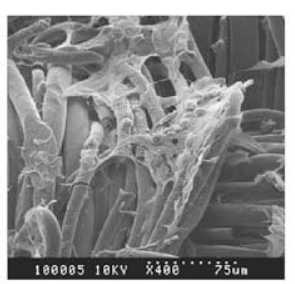

Collagen $(+)$
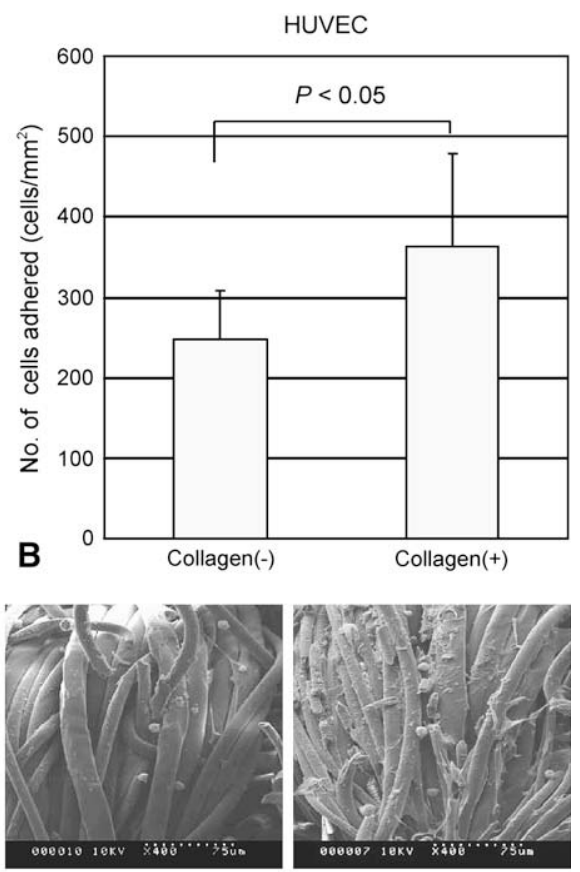

D Collagen(-)
Figure 2. The number of cells of NIH3T3 (A) and human umbilical vascular endothelial cells (B) cultured for 3 days in vitro on TEVGs with/without the collagen microsponge. Scanning electron micrographs of the same samples with NIH3T3 (C) and human umbilical vascular endothelial cells (D) cultured for 3 days in vitro. HUVEC, Human umbilical vascular endothelial cell. 

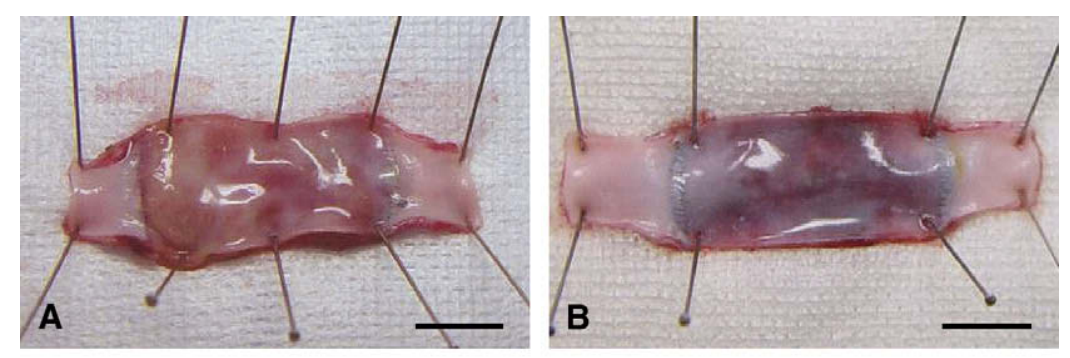

Figure 3. Macroscopic appearance of TEVGs 2 (A), 4 (B), 6 (C), and 12 (D) months after implantation and of the ePTFE graft 12 months after implantation (E). The scale bar represents $10 \mathrm{~mm}$.
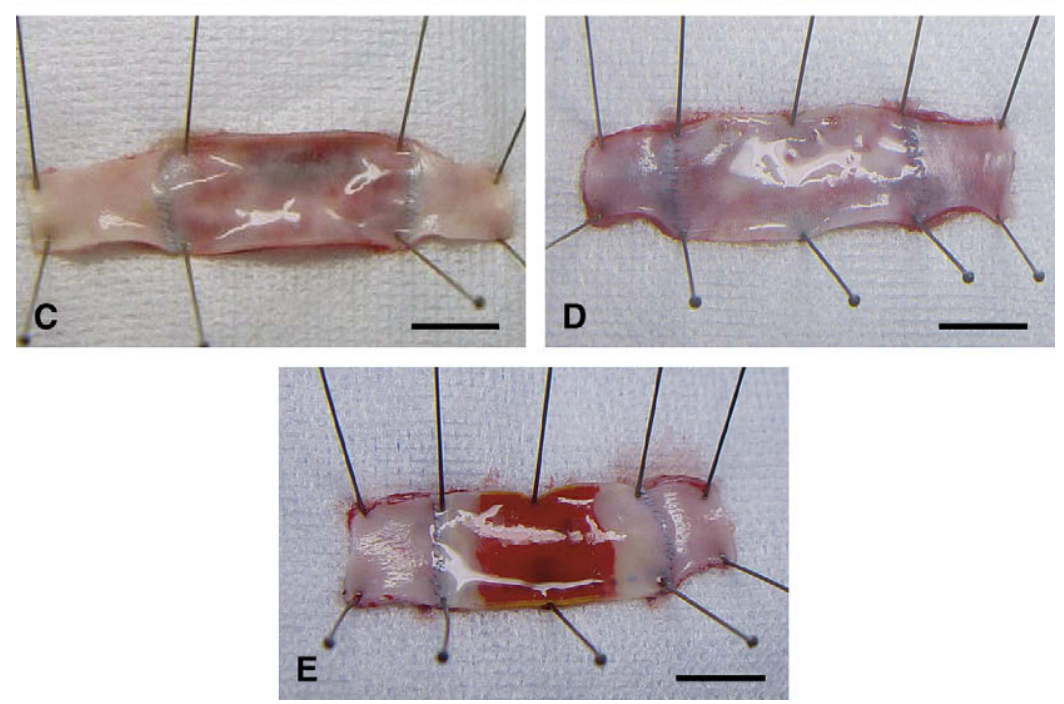

and colleagues ${ }^{12}$ and a standard curve from trans-4-hydroxyproline (Sigma, St Louis, Mo). The measured amounts of hydroxyproline in the samples were compared with those in the native carotid arteries $(\mathrm{n}=16)$.

\section{Biomechanical Examination}

The mechanical properties of TEVGs and native carotid arteries were evaluated using a tensile testing machine (Tensilon; Orientec, Tokyo, Japan). Longitudinal strips measuring $20 \times 5 \mathrm{~mm}$ each were placed within the clamps of the tensile tester and pulled circumferentially to failure at a rate of $10 \mathrm{~mm} / \mathrm{min}$. The tensile force and displacement were recorded, and the tensile strength and modulus were calculated.

\section{Statistical Analysis}

The results of quantitative studies were expressed as the mean \pm standard deviation and analyzed using a statistical analysis software package (Stat-View version 5.0; Abacus Concepts Inc, Berkeley, Calif).

A standard $t$ test was used to determine the significance of the difference between the 2 means, and the appropriate analysis of variance and Tukey-Kramer's post hoc test were used to compare the measured data among multiple groups. $P$ values of less than .05 were considered to be statistically significant.

\section{Results}

\section{In Vitro Study of Cell Culture Growth}

Three days after implantation (experimental day 3), scanning electron microscopy images showed that NIH3T3 and human umbilical vascular endothelial cells were more closely adherent to and more expanded in TEVGs with the collagen sponge than in those without the collagen sponge (Figure 2, $C$ and $D$ ). The cell count test with both cell lines indicated that the number of proliferated cells attached to TEVGs with the collagen microsponge was significantly large, compared with TEVGs without the collagen microsponge (Figure 2, $A$ and $B$ ).

\section{In Vivo Study of Implantation in Mongrel Dogs}

All surgeries were completed successfully, and all animals remained in good health. No dogs presented with signs of local or systemic infection during the entire observation period until they were sacrificed.

\section{Morphologic Examination}

At the time of graft explantation, there was no unusual adhesion, perigraft hematoma, perigraft seroma, or inflammatory reaction. There was no gross evidence of stenosis, aneurysm, or suture dehiscence at any point of time. The luminal surfaces of all 32 explanted TEVGs were smooth and shiny with no evidence of thrombosis (Figure 3, $A-D$ ). The anastomotic sites were smooth and not stenosed. Macroscopically, the surfaces were completely endothelialized. In ePTFE grafts, however, the luminal surfaces were not completely endothelialized at any time (Figure $3, E$ ), and 2 grafts were occluded 12 months after implantation. Scanning electron 

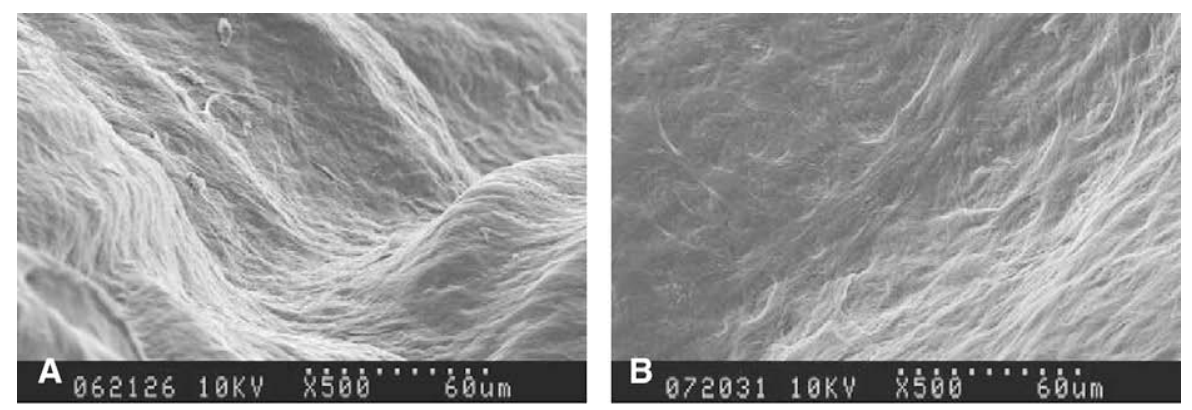

Figure 4. Scanning electron micrograph of the luminal surfaces of TEVGs 2 (A), 4 (B), 6 (C), and 12 (D) months after implantation, compared with that of the ePTFE graft 12 months after implantation (E) (magnification, $500 \times$ ).
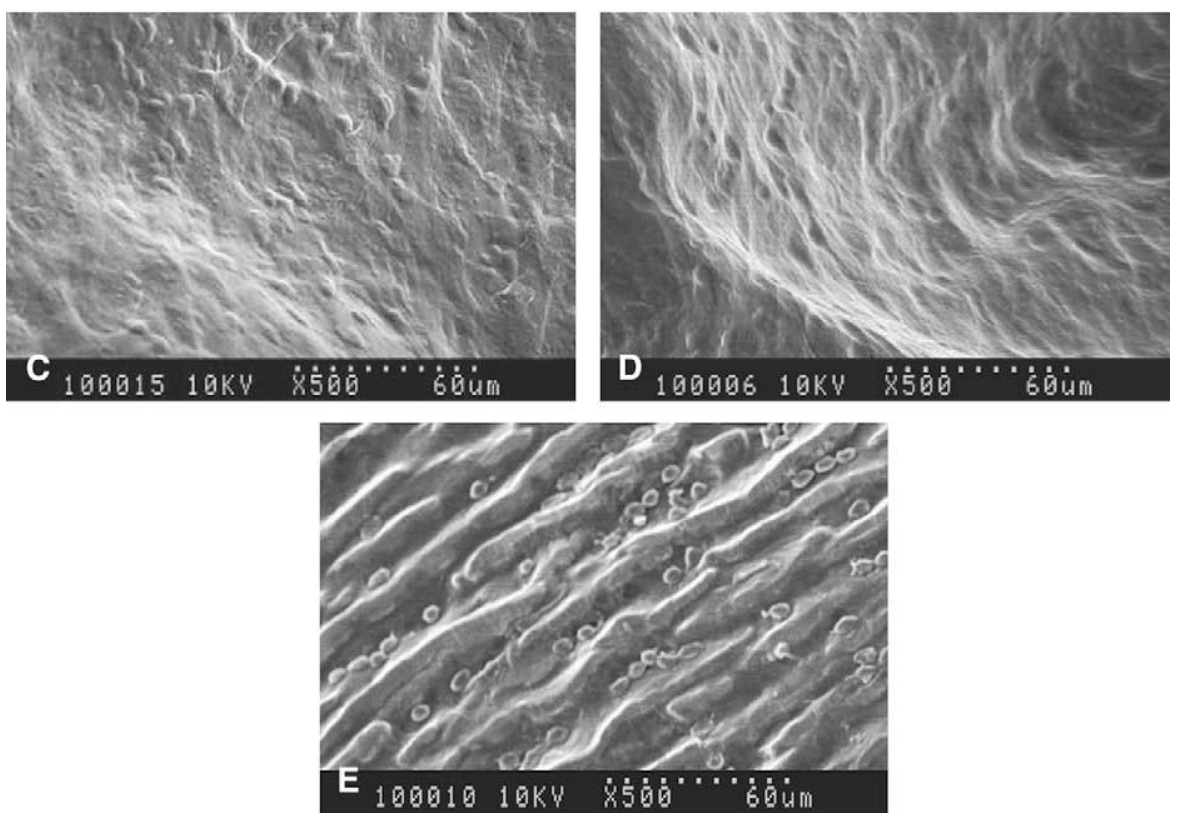

microscopy showed that the luminal surfaces of TEVGs were overlaid with confluent endothelial cells oriented parallel to the direction of the blood flow (Figure 4), and a cross-section of TEVGs showed that PGA fibers were completely absorbed as early as 2 months after implantation; however, PLLA fibers remained unabsorbed throughout the entire study (data not shown).

\section{Histologic and Immunohistologic Examinations}

The explanted TEVGs had a 3-layered structure at all points of time, which was reminiscent of the natural arterial structure (Figure 5). The luminal surface of the newly formed tissue (particularly the intima) showed a complete endothelialization with a confluent endothelial layer consisting of von Willebrand factor-positive cells. The central layer (essentially, the media) was composed of parallel-aligned $\alpha$-smooth muscle actin positive smooth muscle cells and residual scaffold material. The extracellular matrix (ECM) was stained by Victoria blue and elastica van Gieson. The intensity of staining of multilayered elastin and collagen increased with time, indicating the biosynthesis of elastic and collagen fibers. The outer surface (essentially, the adventitia) was composed of the loose connective tissue. The neointimal and medial wall thickness progressively condensed with time, peaked 6 months after implantation, and tissue regeneration slowed down subsequently. Furthermore, no significant differences were observed in the neointimal or medial wall thickness among the proximal, middle, and distal portions of the graft (Figure 6).

\section{Biochemical Examination}

The 4-hydroxyproline assay showed that the amount of collagen in the neotissue of TEVGs increased with time. Six months after implantation, the collagen content in TEVGs was almost the same as that in the native vascular tissue (Figure 7).

\section{Biomechanical Characterization}

After implantation, the strength of TEVGs and neotissue decreased, and the pliability increased to be almost equivalent to that of the native carotid artery. The mechanical tensile strength of TEVGs and neotissue decreased by one half of the preimplantation values $(\mathrm{n}=8)$ and reached a level that was almost equivalent to that of the native carotid artery. After implantation, the tensile modulus of them also reached a level that was almost equivalent to that of the native carotid artery (Figure 8). 


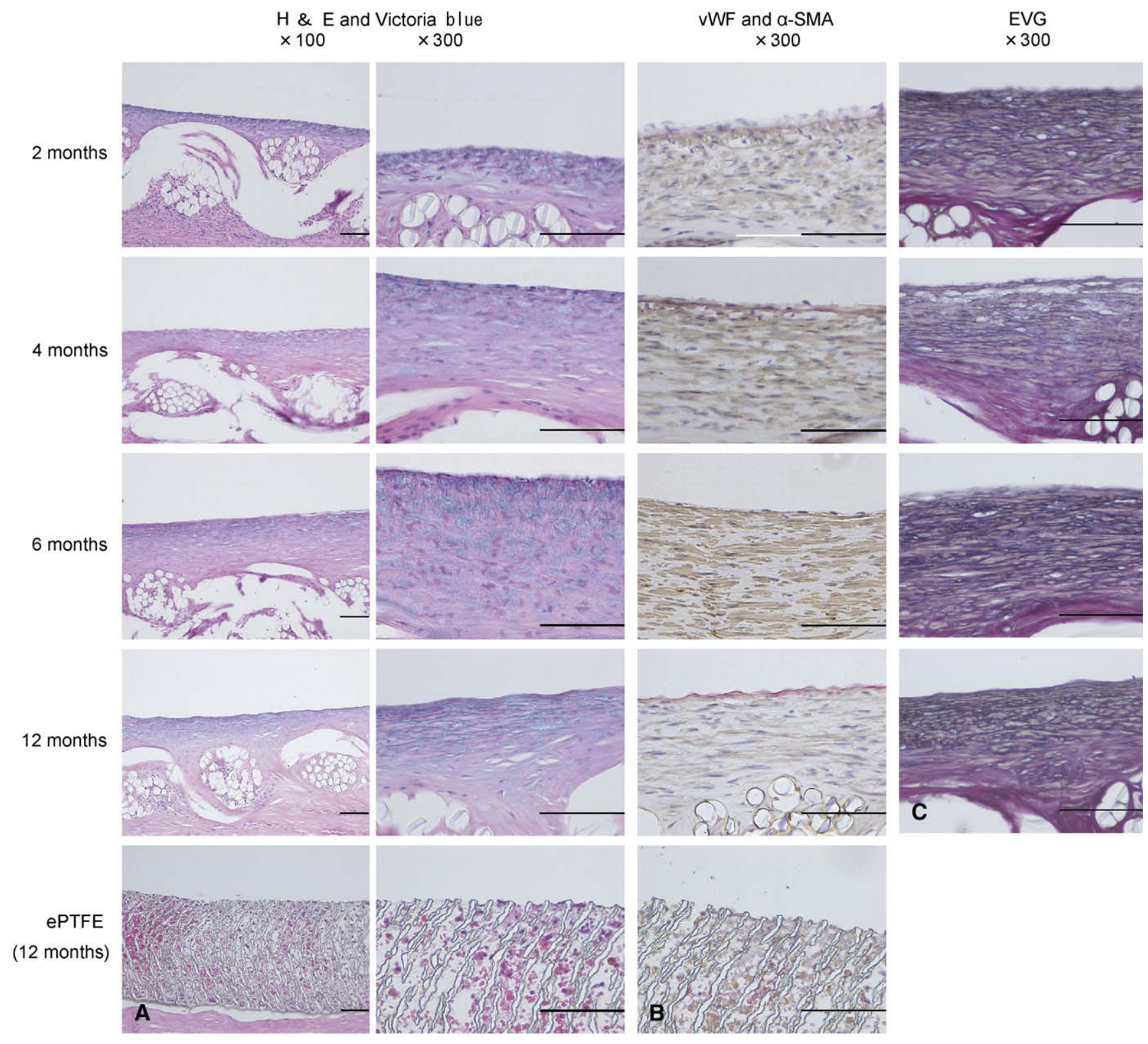

Figure 5. Double staining with hematoxylin-eosin and Victoria blue stain (A), immunohistochemical double staining with von Willebrand factor and $\alpha$-smooth muscle actin (B), and elastica van Gieson (C) of the PGA/PLLA tube graft 2, 4, 6, and 12 months after implantation, and those of the ePTFE graft 12 months after implantation. The scale bar represents $100 \mu \mathrm{m}$. H\&E, Hematoxylin-eosin; vWF, von Willebrand factor; SMA, smooth muscle actin; EVG, elastica van Gieson; ePTFE, expanded polytetrafluoroethylene.

\section{Discussion}

There is a need to develop novel vascular grafts that function well in small-diameter applications because small-caliber vascular grafts are associated with high rates of graft failure. One innovation designed to improve the patency of such grafts is the creation of living autologous replacement materials by methods of tissue engineering. The ultimate goal of tissue engineering is to construct the tissues with characteristics of healthy native tissues from their cellular components. Various types of natural and synthetic scaffolds with arterial tissue cells or differentiated stem cells have attracted much interest as potential small-caliber vascular grafts. However, the manufacturing or pretreatment process of them is complicated, invasive, and time-consuming. It was thought that the synthetic graft that had the potential to promote autologous tissue regeneration without any seeding would be more practical 


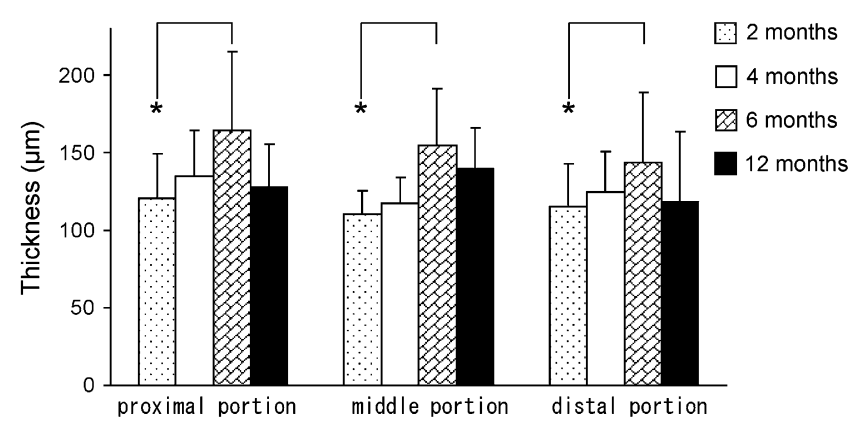

Figure 6. The regenerated neointimal and medial wall thickness of the proximal, middle, and distal portions of TEVGs 2, 4, 6, and 12 months after implantation. Error bars indicate standard deviation. The wall thickness increased significantly over time from 2 to 6 months ( $\left.{ }^{*} P<.05\right)$.

than a seeded graft. In this study, we demonstrated the feasibility of TEVGs in small-diameter artery reconstruction, which were prepared by using a bioengineered material made of collagen microsponge and a biodegradable polymer scaffold. TEVGs used for carotid artery reconstruction in a dog model had a $100 \%$ patency rate with no signs of thrombosis and showed good in situ tissue regeneration for up to 12 months without excessive neointimal hyperplasia or aneurysm formation. These findings suggested that this graft had sufficient durability and patency to be used for small-diameter artery reconstruction.

In our previous study, we prepared a tissue-engineered patch that could promote autologous tissue regeneration without cell seeding and used it for vessel wall reconstruction in large animal models. ${ }^{10}$ This tissue-engineered patch was fabricated by compounding a collagen microsponge with a biodegradable polymer scaffold composed of a PGA-knitted mesh whose outside was reinforced with woven PLLA to avoid rupture and aneurysm formation. In the present study, we slightly changed the design of the polymer scaffold prepared from the same materials to create a small-caliber tube graft. We fabricated the core-sheath compound yarn using

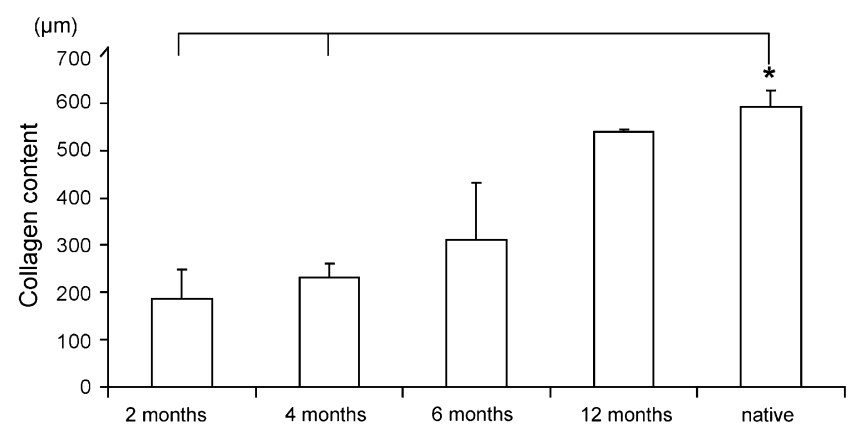

Figure 7. Biochemical analysis of collagen content in the neotissue of TEVGs 2, 4, 6, and 12 months after implantation and that of the native carotid artery $\left({ }^{*} P<.05\right)$.
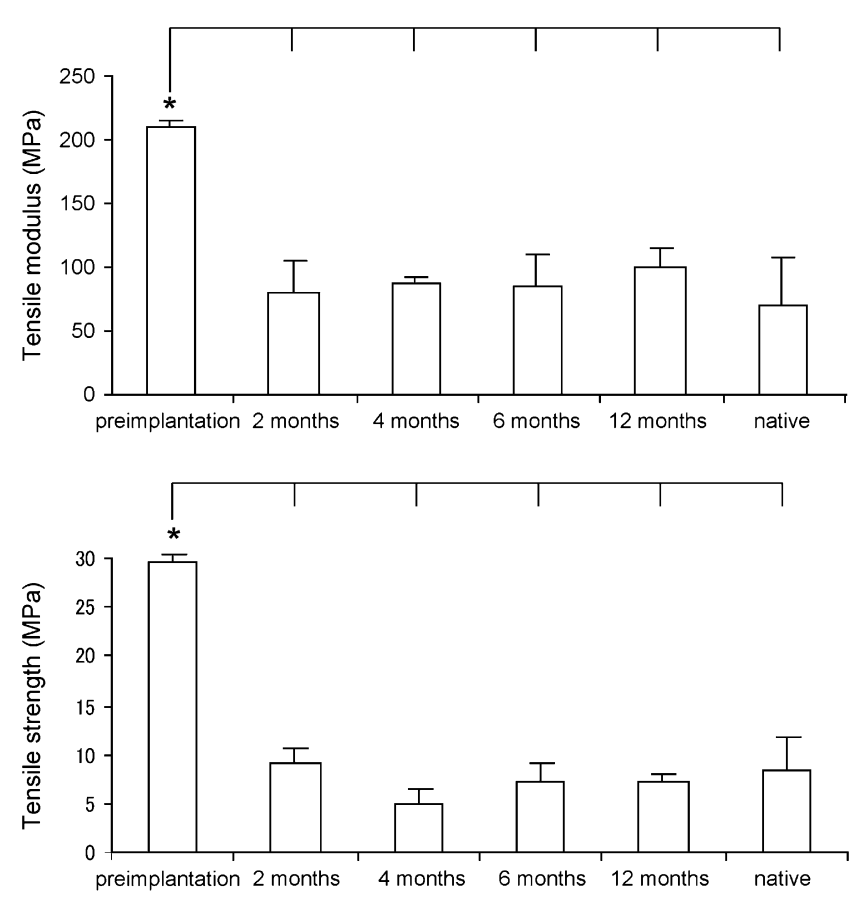

Figure 8. Biomechanical analysis showing tensile modulus and tensile strength of TEVGs at preimplantation stage and 2, 4, 6, and 12 months after implantation, and those of the native carotid artery $\left({ }^{*} P<.05\right)$. MPa, Megapascal.

PGA and PLLA fibers and constructed the small-diameter tube graft in a plain weave pattern. The core-sheath yarn composed of PLLA and PGA was fabricated by air-jet spinning technology and had a porous 3-dimensional structure. Cell culture in vitro showed that proliferation of the cells seeded onto TEVGs with the collagen microsponge was significantly higher than that onto TEVGs without the collagen microsponge. This suggested that the porous 3-dimensional structure and the collagen microsponge could provide a feasible microenvironment to facilitate the in vivo attachment and expansion of autologous cells throughout TEVGs, even in the absence of cell seeding.

Histologically, TEVGs had a 3-layered structure at all points of time, which was reminiscent of the natural arterial structure. The luminal surface of the newly formed tissue (particularly the intima) showed complete endothelialization with a confluent endothelial layer consisting of von Willebrand factor-positive cells. The endothelialization of TEVGs was completed by at least 2 months after implantation. Endothelial cells actively inhibit thrombosis and intimal hyperplasia, and serve as an anticoagulant surface. ${ }^{13,14}$ Because of complete endothelialization, TEVGs were free from thrombosis during the observation period, despite no antiplatelet or anticoagulant drugs being used. In ePTFE grafts, the direct exposure of ePTFE fibrils to the graft lumen and the irregularity of the graft surface at defective sites might cause local 
turbulence in the intraluminal blood flow, resulting in an incidental thrombotic event. The central layer (essentially the media) was composed of multilayered ECM alternating with parallel-aligned $\alpha$-smooth muscle actin positive smooth muscle cells and residual scaffold materials. The outer surface (essentially the adventitia) was composed of the loose connective tissue. This process resembled a prototypical vascular healing response but became progressively condensed at longer intervals. The wound healing response to vascular injury often evolves into a chronic condition of hyperproliferation. Indeed, most studies on synthetic vascular prostheses report massive intimal thickening and pathologically increased matrix synthesis, which results in early graft occlusion and failure. ${ }^{15}$ Therefore, we speculated that this process was indicative of an in situ tissue remodeling process comparable with healing. The results of neointimal and medial wall thickness showed that in situ tissue regeneration in TEVGs by host cells peaked 6 months after implantation and slowed down subsequently. We thought that tissue regeneration stabilized as soon as the process of graft remodeling was completed. In this study, the precise mechanisms behind in situ regeneration have not been evaluated. We can assume that the source of intimal cells is not the anastomotic edges, because a graft of $30 \mathrm{~mm}$ in length may prevent the migration of the cells from the native aorta. ${ }^{12}$ We speculated that the source was the fallout of circulating vascular progenitor cells followed by their differentiation under flow conditions. Overall, it seemed that TEVGs would be a more suitable microenvironment for tissue regeneration by supplying an in situ bioreactor and incubator for vascular progenitor cells without formation of the hyperplastic tissue.

After complete regeneration of the neotissue, the collagen content in its ECM layers was almost the same as that in the native vascular tissue. Instead of collagen, significantly fewer elastic fibers and a thinner elastic layer were observed in the neotissue than in the native aorta; however, the elastic fibers gradually became distinct. Biomechanical examination showed that, after implantation, the strength of TEVGs and neotissue decreased and that the pliability increased to almost the same level of those of the native artery. We speculated that ECM in the neotissue might withstand the aortic blood pressure, although the strength of TEVGs reduced after grafting because of hydrolysis and biological processes. Moreover, arterial pulsation and transmission of shear stress to the graft wall might not be prevented, although the TEVG scaffold remained intact.

The limitations of our study were the residual PLLA fibers in the TEVG scaffold, and the graft size was considerably large for clinical use. To withstand the aortic pressure, PLLA was used for preparing the graft scaffold. However, residual PLLA was noted until 12 months after implantation. It was necessary to investigate whether the newly formed tissue could withstand the aortic blood pressure even after complete degradation of the graft scaffold; therefore, further long-term follow-up was required. We needed to optimize the degradation time of the scaffold material, because the cellular structures and matrix were absorbed, until only the engineered tissues remained without any foreign materials to withstand the aortic pressure. For the next step, we should consider the smaller and longer grafts for established use in grafting procedures involving small-diameter vessels, such as the peripheral arteries. Nevertheless, we believe that in situ tissue regeneration using TEVGs that do not require pretreatment in the form of cell seeding may be a useful strategy and may provide further experimental justification for entering clinical trials.

\section{Conclusions}

Our study indicated that a novel tissue-engineered, smallcaliber, biodegradable vascular graft promoted in situ vascular tissue regeneration and did not require pretreatment in the form of cell seeding, thereby conferring better patency on small-diameter vascular grafts.

We acknowledge the excellent technical assistance of Shigeru Matsunui and Tadashi Mukouyama.

\section{References}

1. Langer R, Vacanti JP. Tissue engineering. Science. 1993;260:920-6.

2. Pasoc M, Muller-Glauser W, Von Segesser LK, Lanchat M, Mihaljevic T, Turina MI. Superior late patency of small-diameter Dacron grafts seeded with omental microvascular cells: an experimental study. Ann Thorac Surg. 1994;58:677-83.

3. Kaube HR, Duwe J, Rutsch W, Konertz W. Clinical experience with autologous endothelial cell-seeded polytetrafluoroethylene coronary artery bypass grafts. J Thorac Cardiovasc Surg. 2006;120:134-41.

4. Niklason LE, Gao J, Abbott WM, Hirschi KK, Houser S, Marini R, et al. Functional arteries grown in vitro. Science. 1999;284:489-93.

5. Hoerstrup SP, Cummings I, Lachat M, Schoen FJ, Jenni R, Leschka S, et al. Functional growth in tissue-engineered living, vascular grafts. Follow-up at 100 weeks in a large animal model. Circulation. 2006;114:I-159-66.

6. Kaushal S, Amiel GE, Guleserian KJ, Shapira OM, Perry T, Sutherland FW, et al. Functional small-diameter neovessels created using endothelial progenitor cells expanded ex vivo. Nat Med. 2001;7: 1035-40.

7. Shin'oka T, Imai Y, Ikeda Y. Transplantation of a tissue engineered pulmonary artery. $N$ Engl J Med. 2001;344:532-3.

8. Hibino N, Shin'oka T, Matsumura G, Ikeda Y, Kurosawa H. The tissue engineered vascular graft using bone marrow without culture. J Thorac Cardiovasc Surg. 2005;129:1064-70.

9. Shin'oka T, Matsumura G, Hibino N, Naito Y, Watanabe M, Komuro T, et al. Midterm clinical result of tissue engineered vascular autografts seeded with autologous bone marrow cells. J Thorac Cardiovasc Surg. 2005;129:1330-8.

10. Iwai T. Novel tissue-engineered biodegradable material for reconstruction of vascular wall. Ann Thorac Surg. 2005;80:1821-8.

11. Chen G. A biodegradable hybrid sponge nested with collagen-microsponges. J Biomed Mater Res. 2000;51:273-9.

12. Huzer G, Maiocco J, Naftolin F. Monitoring of collagen and collagen fragments in chromatography of protein mixtures. Anal Biochem. 1980;105:424-9.

13. Davie EW. Biochemical and molecular aspects of the coagulation cascade. Thromb Haemost. 1995;74:1-6.

14. Gimbrone MA Jr, Bevilacqua MP, Cybulsky MI. Endothelial-dependent mechanisms of leukocyte adhesion in inflammation and atherosclerosis. Ann NY Acad Sci. 1990;598:77-85.

15. Ao PY, Hawthorne WJ, Vicaretti M, Fletcher JP. Development of intimal hyperplasia in six different vascular prostheses. Eur J Vasc Endovasc Surg. 2000;20:241-9. 